\title{
SCIDoC
}

\author{
International Journal of Dentistry and Oral Science (IJDOS) \\ ISSN: 2377-8075
}

\section{Speech Effect Of Fixed Labial Orthodontic Appliance In Patient With Class III Incisor Relationship}

Research Article

\author{
Khan $\mathrm{KAM}^{1 *}$, Law ${ }^{1}$, Badrulzaman ${ }^{2}$
}

${ }^{1}$ Discipline of Orthodontics, Department of Family Oral Health, Faculty of Dentistry, University Kebangsaan Malaysia, Malaysia.

${ }^{2}$ Centre for Rehabilitation and Special Needs, Faculty of Health Sciences, University Kebangsaan Malaysia, Malaysia.

\section{Abstract}

Objective: To evaluate the pre-existing speech distortion and long-term speech effect of the fixed labial orthodontic appliance in Class III incisor relationship (Class III) subjects.

Methods: Speech performance of selected fricative consonants was analyzed with PRAAT software inClass I incisor relationship (Class I control) subjects as the baseline as well as in Class III subjects at six different time-points.

Results: Class III subjects took shorter voicing duration to utter all sounds at T0 compared to Class I control subjects. At T1, Class III subjects took longer voicing duration to utter the sounds compared to T0. No significant difference was found between T4 and T0. At T5, no significant difference was found between Class III subjects and Class I control subjects. Conclusions: Class III subjects had significant pre-existing speech distortion and further deterioration in the speech quality was reported immediately after bracket placement which self-resolved after 6 months. Orthodontic treatment improved the pre-existing speech distortion in Class III subjects after a year.

Keywords: Speech; Class III Malocclusion; Fixed Labial Orthodontic Appliance.

\section{Introduction}

Individuals with Class III incisor relationship presented with a reduced overjet or edge-to-edge incisor relationship to reversed overjet depending on the severity of the underlying skeletal discrepancy. The incidence of Class III incisor relationship is estimated to be $3-5 \%$ in the Caucasian population (Foster \& Day, 1974) and $4-13 \%$ in the Oriental population (Irie \& Nakamura, 1975). The Class III incisor relationship individuals were found to have difficulty in articulation of labiodental and alveolar fricative consonants which were produced by constriction or closure at some point along the vocal tract (Guyer et al., 1986; Laine, 1987; Laine, 1992). Therefore, a deviation in dental structure or alignment may interfere with airflow and pressure and there by alter speech sound productionin which teeth position may contribute $50 \%$ to $60 \%$ of all speech disorder (Van Riper, 1978).

Apart from that, the orthodontic treatment itself is associated with the adverse effect of speech distortion (Papageorgiou et al., 2016; Long et al., 2013) and it results in a significant negative impact on patients' compliance as well as the quality of life. The speech distortion arises as a result of the affected movement of tongue and space of an oral cavity by an orthodontic appliance which is fitted against the teeth surface and palate (Rai et al., 2014). Studies found that orthodontic appliances such as labial and lingual fixed appliance (Rai et al., 2013; Khattab et al., 2013; Rai et al., 2014) and retainer (Atik et al., 2017; Wan et al., 2017) will cause speech distortion. It had been reported that the irregularity in the anterior region of the mouth can cause tongue protrusion and affect the articulation of /s/ sound (Paley et al., 2015). The duration of speech disturbance caused by the fixed labial appliance is variable and the difference may be attributed to the individual adaptation as well as the severity of the malocclusion (Paley et al., 2015).

Even though speech distortion can cause a serious barrier in social and professional functioning (Millard \& Richman, 2015), there

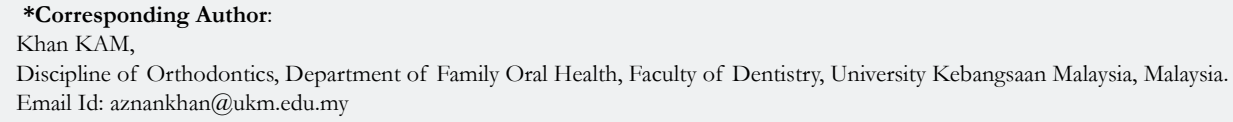

Copyright: Khan $\mathbf{K A M}^{\circ}$ 2021. This is an open-access article distributed under the terms of the Creative Commons Attribution License, which permits unrestricted use, distribution and reproduction in any medium, provided the original author and source are credited. 
is a lack of evidence on the long-term effect of the fixed labial orthodontic appliance on speech sound production. Therefore, studies focusing on the speech impact of a specific orthodontic appliance in specific malocclusion are warranted to allow the orthodontist to provide adequate information regarding speech, so that patients can make an informed decision and be prepared to adapt to the potential speech problems accordingly. This prospective controlled study aimed to evaluate the long-term effect of the fixed labial orthodontic appliance on speech in an individual with Class III incisor relationship.

\section{Objectives}

The main objectives of the study were:

To compare the baseline speech quality between Class III subjects and Class I control subjects.

To describe the changes of speech sound in Class III subjects after placement of fixed labial orthodontic appliance for up to a year.

To determine the impact of overjet correction in Class III subjects on speech sound quality.

It was hypothesized that Class III subjects have significant preexisting speech distortioncompared to Class I control subjects, the fixed labial orthodontic appliance placement will lead to further speech distortion in short-term and corrective orthodontic therapy will improve the pre-existing speech.

\section{Materials and Methods}

\section{Subjects}

Ethical approval was obtained from the Research \& Ethics Committee, Hospital University Kebangsaan Malaysia. Sample size calculation was done using Sample Size Calculator (CTSI/UCSF) for before-after study (Paired t-test) with a Type I error rate of 0.05 , Type II error rate of 0.20 , an effect size of 0.70 , and standard deviation of change valueof 1.00 (Khan et al., 2017). This generated a total sample size of sixteen subjects for this study. The target sample size is increased to twenty subjects in allowing a possible dropout rate of $30 \%$. Twenty Class III incisor relationship (Class III) subjects satisfying the inclusion and exclusion criteria were recruited in the study. The inclusion criteria were subjects indicated for fixed labial orthodontic appliance treatment, Class III incisor relationship subjects indicated for dento alveolar compensation, subjects who were local citizens and attending or attended national school, and subjects with age more than fourteen years old
(Kent \& Read, 1992). The exclusion criteria were subjects with the craniofacial defect such as cleft lip and palate or syndromic, subjects with hearing and speech disorder, and subjects who had previous orthodontic treatment. Besides, Class I incisor relationship (Class I control) subjects were number and age-matched with Class III subjects. All subjects were explained about the study and consents were obtained from the subjects or the guardians before being recruited.

\section{Objective Evaluation}

Speech performance analysis was done for Class I control group as the baseline by using a list of fricative consonant words that are most susceptible to articulation error including 'foto, video, sapu, zaman'. Recordings were conducted in a standardized condition using a Sony® ICD-SX2000RC Digital Voice Recorder in a quiet room and at a distance of $10 \mathrm{~cm}$ from the subjects' mouth. Three recordings of each fricative consonant / $\mathrm{f}, \mathrm{v}, \mathrm{s}, \mathrm{z} /$ were recorded and analyzed with PRAAT software (www.praat.org) by measuring the voicing duration of the fricative consonant in milliseconds (ms) on the generated spectrographas shown in Figure 1. PRAAT software is a valid tool used in multiple orthodontic speech studies (Rai et al., 2013; Rai et al. 2014; Wan et al., 2017; Khan et al., 2017).

For Class III subjects, the speech performance analysis was done in the same standardized protocolmentioned earlier before the placement of the fixed labial orthodontic appliance at T0. Thereafter, Class III subjects underwent a standardized bond up process at which the upper and lower teeth for each subject were cleaned with pumice, etched, and followed by bonding with Preadjusted edgewise fixed labial orthodontic appliances with MBT prescription, 0.022x 0.028-inch slot size (American Orthodontics) for both arches. The 0.014-inch nickel-titanium wires were used as initial archwire and secured with elastomeric modules. The subjects were reviewed monthly and treated according to the four phases of orthodontic camouflage treatment which were leveling and alignment, over bite control, space closure, and finishing. The standardized speech performance analysis mentioned earlier was repeated at five different time-points which were with in 24 hours (T1), 1 month after (T2), 3 months after (T3), 6 months after (T4), and 1 year after (T5) placement of fixed labial orthodontic appliance. Besides, overjet was measured in millimeters $(\mathrm{mm})$ at three different timepoints which were T0, T4, and T5 in Class III subjects.

\section{Method Error Analysis}

To ensure the consistency of the voicing duration measurements, three recordings were analyzed with the PRAAT software for each subject at each timepoint and the average values were taken as

Figure 1. A spectrograph displaying the word 'foto'. The highlighted portion indicates the voicing duration of the fricative consonant /f/ sound.

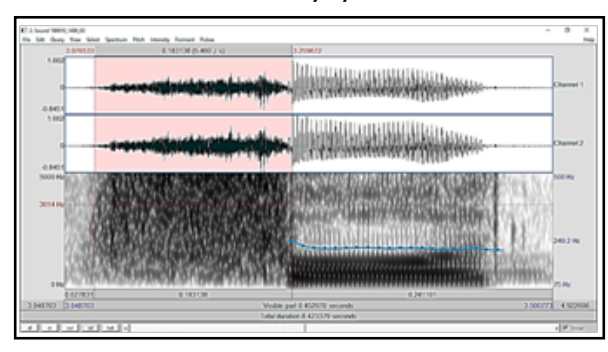


the final readings. Besides, 200 speech samples were re-measured two weeks after the initial measurements and an intra-examiner reliability test was performed using the Intra-class Correlation Coefficient test (ICC) between the initial and the repeated measurements. There was a significant correlation between the measurements with an $r>0.99$. This suggested that there was excellent intra-examiner reliability in the measurement of the voicing durations. Apart from that, the consistency of the overjet measurement was monitored as well in which 10 samples of overjet were re-measured two weeks after the initial measurements. A significant correlation between the measurement with an $r>0.98$ was found by using the Intra-class Correlation Coefficient. This again suggested that there was excellent intra-examiner reliability in the measurement of the overjet.

\section{Statistical Analysis}

Statistical analysis was done using Statistical Package for Social Science (SPSS) software version 23.0. The normality of the data was checked with the Shapiro-Wilk normality test. Independent ttest or Mann-Whitney $\mathrm{U}$ test was used to compare the difference in voicing duration for each sound and the overjet difference between Class III subjects and Class I control subjects at T0 and T5. One-way repeated ANOVA was used to compare the mean voicing duration of each phone me at different time points in Class III test subjects. Paired sample t-test with Bonferroni adjusted $\alpha$ of 0.08 was used to compare the mean voicing duration of each phoneme in Class III subjects before (T0) and after placement of the fixed labial orthodontic appliance at different time-points as well as the overjet changes at T0, T4, and T5.

\section{Results}

\section{Subjects Flow and Baseline Data}

Twenty Class III subjects satisfying the inclusion and exclusion criteria were recruited at the beginning of the study. There was one drop out of the twenty subjects due to the inability to comply with speech recordings at different time-points, hence nineteen Class III subjects remained. The age range of Class III subjects was from 14 years old to 33 years old with a mean of 22.4 years old. The mean initial overjet of Class III subjects was $0.2 \mathrm{~mm}$ and ranged from $-2.0 \mathrm{~mm}$ to $1.5 \mathrm{~mm}$ as shown in Table 1 . Thirteen of the subjects were female and seven of them were male. Nineteen Class I incisor relationship subjects with mean overjet of $3 \mathrm{~mm}$ and a median age of 23 years old were recruited as the Class I control group.

\section{Objective Evaluation}

Class III subjects took a shorter voicing duration to utter the fricative consonants at T0 compared to the Class I control subjects as shown in Table 2.

One-way repeated measures ANOVA analysis showed that there were significant changes in the mean voicing duration (in milliseconds) for each sound at various time-points in Class III subjects shown in Table 3. Table 4 shows the changes in the mean voicing duration for each sound at each time-point by comparing the mean voicing duration of each sound at each timepoint after insertion of a fixed labial orthodontic appliance with the mean voicing duration before insertion of a fixed labial orthodontic appliance with paired sample t-test, T0. It shows that the voicing duration for each sound was statistically significant increased at T1, T2, and T3 compared to T0. At T4, the voicing duration for each sound was reduced and became statistically insignificantly different from the value at $\mathrm{T} 0$ as shown with the $\mathrm{p}$-valueof more than 0.05 for all the sound at T4. At T5, however, the voicing duration for each sound appeared to be statistically significant increased again in comparison to the voicing duration at T0.

Table 5 shows the statistical difference of overjet and voicing du-

Table 1. Comparison of voicing duration \pm standard deviation (SD) in milliseconds (ms) of sounds between Class III test subjects at T0 with Class I control subjects.

\begin{tabular}{|c|c|c|c|}
\hline \multirow[b]{2}{*}{ Sounds } & \multicolumn{2}{|c|}{ Voicing duration \pm SD (ms) } & \multirow[b]{2}{*}{ p-value } \\
\hline & $\begin{array}{l}\text { Class III test } \\
\text { subjects }\end{array}$ & $\begin{array}{l}\text { Class I con- } \\
\text { trol subjects }\end{array}$ & \\
\hline$/ \mathrm{f} /$ & $122.5 \pm 16.4$ & $192.6 \pm 15.5$ & $0.000^{*}$ \\
\hline$/ \mathrm{v} /$ & $76.1 \pm 23.2$ & $131.5 \pm 15.5$ & $0.000^{*}$ \\
\hline /s/ & $151.8 \pm 27.9$ & $188.2 \pm 10.6$ & $0.000^{*}$ \\
\hline $\mid z /$ & $96.7 \pm 21.5$ & $141.2 \pm 14.2$ & $0.000^{* *}$ \\
\hline
\end{tabular}

Asterisk $\left({ }^{\top}\right)$ indicated the statistical significance of the Mann-Whitney $\mathrm{U}$ test; $\left(^{*}\right)$ indicated statistical significance for the independent t-test.

Table 2. The F statistics with p-values and mean voicing duration \pm standard deviation (SD) in milliseconds (ms) of the sounds.

\begin{tabular}{|c|c|c|c|c|c|c|c|}
\hline \multirow{2}{*}{ Sounds } & \multicolumn{6}{|c|}{ Mean voicing duration $\pm \mathrm{SD}(\mathrm{ms})$} & \multirow{2}{*}{ p-value } \\
\hline & T0 & T1 & $\mathrm{T} 2$ & T3 & T4 & T5 & \\
\hline$/ f /$ & $122.5 \pm 16.4$ & $184.6 \pm 28.7$ & $178.7 \pm 34.0$ & $183.9 \pm 36.7$ & $129.3 \pm 13.7$ & $183.1 \pm 11.5$ & $<0.001^{*}$ \\
\hline$/ \mathrm{v} /$ & $76.0 \pm 23.2$ & $115.2 \pm 39.5$ & $124.4 \pm 42.4$ & $123.1 \pm 40.0$ & $82.5 \pm 14.7$ & $131.6 \pm 8.9$ & $<0.001^{*}$ \\
\hline$/ \mathrm{s} /$ & $151.8 \pm 27.9$ & $162.6 \pm 33.6$ & $184.1 \pm 31.2$ & $184.6 \pm 34.5$ & $147.8 \pm 29.9$ & $187.3 \pm 12.0$ & $<0.001^{*}$ \\
\hline $\mid z /$ & $96.7 \pm 21.5$ & $108.1 \pm 25.8$ & $130.0 \pm 28.0$ & $130.8 \pm 36.4$ & $101.8 \pm 29.6$ & $139.2 \pm 10.7$ & $<0.001^{*}$ \\
\hline
\end{tabular}


ration for each sound of Class III subjects at T0, T4, and T5 compared to Class I control subjects. It was found that the mean overjet between Class III subjects at T0, T4, and T5 was $0.2 \mathrm{~mm}$, $1.0 \mathrm{~mm}$, and $2.5 \mathrm{~mm}$ respectively which was significantly lower compared to Class I control subjects with an average overjet of $3 \mathrm{~mm}$. However, the differences in overjets of Class I control subjects with a mean of $3 \mathrm{~mm}$ and Class III subjects with a mean of $2.5 \mathrm{~mm}$ at T5 were clinically insignificant, although they were shown to be a statistically significant difference with the $\varrho<0.05$. Besides, it demonstrated that the mean voicing duration for all sounds in Class III subjects at T0 and T4 was significantly different compared to Class I control subjects. At T5, however, the voicing duration for each sound appeared to become comparable compared to the voicing duration of Class I control subjects.

\section{Discussion}

\section{Main Findings}

The age of the subjects was set to be above 13 years old as one of the inclusion criteria because speech sound acquisition was usually complete after the age of 11 or 12 years old (Kent \& Read, 1992). Hence, the selection of subjects above the age of 13 years old allowed the elimination of possible confounding factors due to incomplete speech sound development. Although one may be argued that gender inequality may cause potential bias in this study, that is the normal gender treatment ratio in the orthodontic clinic since female tends to be more dissatisfied with dental appearance compared to male resulting in higher orthodontic treatment demand in the female (Shaw, 1981).

It was found that Class III subjects took significantly shorter voicing duration to utter all the four fricative consonants at $\mathrm{T} 0$ which consisted of labiodental such as /f, v/ and alveolar such as /s, z/ fricative consonants compared to Class I control subjects. That was because of altered speech articulation as a result of reduced overjet in which the initial presenting mean overjet in Class III subjects was $0.2 \mathrm{~mm}$ at T0 compared to the baseline of $3 \mathrm{~mm}$ forClass I control subjects. The reduced voicing duration caused significant speech distortion in Class III subjects (Laine, 1987, 1992; Guay et al., 1978; O’Gawa \& Wilson, 2007).

Distinctive patterns of changes in voicing duration for the fricative consonants were observed in Class III subjects after the insertion of the fixed labial appliance. The voicing duration for all the sounds increased significantly at $\mathrm{T} 1$ compared to $\mathrm{T} 0$ and fluctuated minimally at $\mathrm{T} 2$ and $\mathrm{T} 3$. The increased voicing duration after placement of the fixed labial orthodontic appliance indicated speech distortion. These findings were supported by studies which stated that orthodontic treatment is associated with significant distortion in speech (Papageorgiou et al., 2016; Long et al., 2013). Distortion of specific sound occurs as a result of the disturbing movement of tongue and space in the oral cavity by an orthodontic appliance which was fitted against the teeth surface (Rai et al., 2014). The most affected sounds were found to be vowels such as /i/, /u/ and /a/ and consonants such as /s/, /t/, /f/ and /1/ (Rai et al., 2013; Rai et al., 2014; Khattab et al., 2012). At T4, however, the voicing duration in Class III subjects for all the sounds seem to be significantly reduced and became statistically insignificant different compared to T0. These findings showed that there wasa significant improvement in speech after 6 months oftreatment due to adaptation to the fixed labial orthodontic appliance by the surrounding articulating organs such as lips, tongue, cheek, and muscles (Rai et al., 2013; Rai et al., 2014; Khattab et al., 2012; Paley et al., 2015).

Table 3. Mean difference of voicing duration \pm standard deviation (SD) in milliseconds (ms) for each sound at different time points in comparison with before insertion of fixed labial orthodontic appliance.

\begin{tabular}{|c|c|c|c|c|c|}
\hline \multirow{2}{*}{ Sounds } & \multicolumn{5}{|c|}{ Mean difference of voicing duration \pm SD (ms) } \\
\hline & T1 - T0 & $\mathrm{T} 2-\mathrm{T} 0$ & T3-T0 & T4 - T0 & T5 - T0 \\
\hline$/ \mathrm{f} /$ & $63.1 \pm 29.3^{*}$ & $56.2 \pm 36.7^{*}$ & $61.4 \pm 40.3^{*}$ & $6.7 \pm 18.6$ & $60.1 \pm 20.3^{*}$ \\
\hline$/ \mathrm{v} /$ & $39.2 \pm 33.1^{*}$ & $48.4 \pm 41.4^{*}$ & $47.1 \pm 43.9^{*}$ & $6.6 \pm 27.7$ & $55.6 \pm 23.6^{*}$ \\
\hline$/ \mathrm{s} /$ & $10.8 \pm 17.8^{*}$ & $32.3 \pm 29.5^{*}$ & $32.7 \pm 32.0^{*}$ & $4.1 \pm 23.2$ & $35.4 \pm 22.3^{*}$ \\
\hline $\mid \mathrm{z} /$ & $11.5 \pm 20.1^{*}$ & $36.3 \pm 27.9^{*}$ & $34.1 \pm 42.6^{*}$ & $5.1 \pm 19.2$ & $42.5 \pm 19.0^{*}$ \\
\hline
\end{tabular}

Table 4. Statistical difference of overjet and voicing duration for each sound of Class III test subjects at T0, T4, and T5 compared to the Class I control subjects.

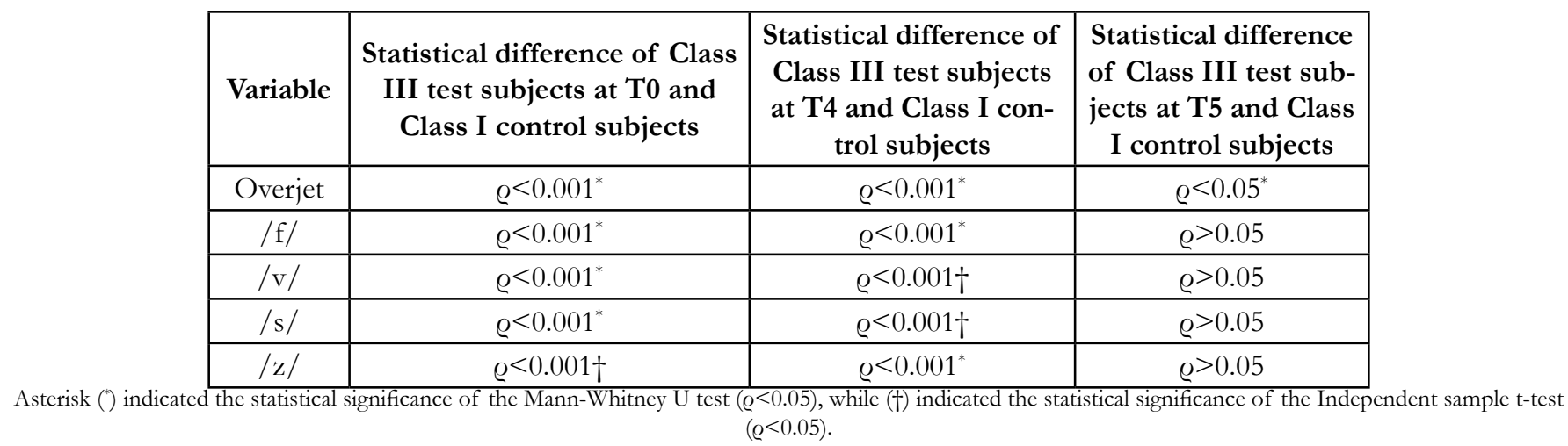


Table 5. Statistical evaluation of the standardized questionnaire.

\begin{tabular}{|c|c|c|c|c|c|}
\hline \multirow[t]{2}{*}{ Question } & \multicolumn{4}{|c|}{ Patients' Response, \% } & \multirow[t]{2}{*}{$P$ (vs T0) } \\
\hline & None & A little & Much & Very much & \\
\hline \multicolumn{6}{|l|}{ Question 1} \\
\hline $\mathrm{T} 2$ & 0 & 21.1 & 26.3 & 52.6 & $<0.001 \dagger$ \\
\hline T3 & 0 & 42.1 & 36.8 & 21.1 & $<0.001 \dagger$ \\
\hline $\mathrm{T} 4$ & 47.4 & 36.8 & 15.8 & 0 & $<0.05 \dagger$ \\
\hline T5 & 63.2 & 36.8 & 0 & 0 & $<0.05 \dagger$ \\
\hline \multicolumn{6}{|l|}{ Question 2} \\
\hline $\mathrm{T} 2$ & 21.1 & 26.3 & 36.8 & 15.8 & $<0.001 \uparrow$ \\
\hline T3 & 36.8 & 42.1 & 21.1 & 0 & $<0.05 \dagger$ \\
\hline $\mathrm{T} 4$ & 63.2 & 36.8 & 0 & 0 & $<0.05 \dagger$ \\
\hline T5 & 84.2 & 15.8 & 0 & 0 & $>0.05$ \\
\hline \multicolumn{6}{|l|}{ Question 3} \\
\hline $\mathrm{T} 2$ & 78.9 & 15.8 & 5.3 & 0 & $<0.001 \dagger$ \\
\hline T3 & 0 & 31.6 & 36.8 & 31.6 & $<0.001 \dagger$ \\
\hline $\mathrm{T} 4$ & 0 & 10.5 & 36.8 & 52.6 & $<0.05 \dagger$ \\
\hline T5 & 0 & 0 & 26.3 & 73.3 & $<0.001 \dagger$ \\
\hline \multicolumn{6}{|l|}{ Question 4} \\
\hline $\mathrm{T} 2$ & 15.8 & 42.1 & 31.6 & 10.5 & $<0.001 \dagger$ \\
\hline T3 & 36.8 & 52.6 & 10.5 & 0 & $<0.05 \dagger$ \\
\hline $\mathrm{T} 4$ & 78.9 & 21.1 & 0 & 0 & $<0.05 \dagger$ \\
\hline T5 & 89.5 & 10.5 & 0 & 0 & $>0.05$ \\
\hline
\end{tabular}

Asterisk $(+)$ indicated the statistical significance of the Wilcoxon signed-rank test analysis $(\varrho<0.05)$.

In the meantime, there were significant differences in the overjet and voicing duration for all the fricative consonants between Class III subjects at T4 and Class I control subjects as shown in Table 5. The difference in overjet was because most of the Class III subjects were still at the stage of leveling and aligning of the pre-adjusted edge wise appliance system in which no significant change in the overjet was found (Scott et al., 2008). Likewise, significant differences in voicing duration for all fricative consonants were found between both groups as a result of the discrepancy in overjet (Laine, 1987, 1992; Guay et al., 1978; O’Gawa \& Wilson, 2007).

It was found that mean differences between Class III subjects and Class I control subjects was statistically significant only for the mean overjet, whilst all the differences in voicing duration were in significant at T5. However, the difference in mean overjet between Class III subjects with a mean of $2.5 \mathrm{~mm}$ and Class I control subjects with a baseline overjet of $3 \mathrm{~mm}$ was clinically insignificant. A significant improvement in the overjet of Class III subjects was noted at T5 because the subjects were at the stage of incisor relationship correction which took place after the stage of leveling and aligning. It could be concluded that as the overjet became normalized in Class III subjects following orthodontic corrective therapy, the voicing duration for all the sounds became normalized as well compared to the voicing duration of Class I control subjects and resulted in normalization of speech in Class III subjects. This finding was supported by studies that found that speech in an individual with defective speech often improved following corrective orthodontic treatment (Rathbone \& Snidecore, 1959; Kirveskari et al., 1988).

\section{Clinical Implication}

The orthodontic treatment has become a routine dental treatment due to the increased demand for aesthetics and function by the general population, especially in the adult population. Hence, there should be a clear list of benefits for the patient and these should over weight any potential risk. This study supports the claim that individuals with Class III incisor relationship exhibit significant speech distortion compared to those with Class I incisor relationship. It provides evidence to acknowledge the complaint of individuals with Class III incisor relationship that there is an associated difficulty in pronunciation of certain sounds, particularly the fricative consonants which are susceptible to irregularities at the anterior teeth. The orthodontists should, therefore, recognize the defective sounds and appreciate the role of dental structures have concerning the pronunciation and articulation of speech.

Besides, patients shall be counseled that wearing the fixed labial orthodontic appliance will lead to speech distortion in the shortterm, but it is temporary and likely to be self-resolved completely up to six months due to adaptation. This information allows patients to prepare themselves emotionally so that they can adjust their social and professional responsibilities accordingly.

Lastly, this study also provides evidence that the correction of reduced or reversed overjet in individuals with Class III incisor relationship will lead to improvement of the pre-existing speech distortion in those individuals. This is a valuable benefit of orthodontic treatment leading to improved quality of life. 


\section{Conclusion}

1. Class III subjects had significant pre-existing speech distortion compared Class I control subjects.

2. The speech further worsens after insertion of a fixed labial orthodontic appliance in Class IIIsubjects and it took up to six months to resolve.

3. The correction of reduced overjet in Class III subjects normalized their pre-existing speech distortion and became comparable with Class I control subjects.

\section{References}

[1]. Foster TD, Day AJ. A survey of malocclusion and the need for orthodontic treatment in a Shropshire school population. Br J Orthod. 1974 Apr;1(3):738. PubmedPMID: 4525734.

[2]. Irie M, Nakamura S. Orthopedic approach to severe skeletal Class III malocclusion. Am J Orthod. 1975 Apr;67(4):377-92. PubmedPMID: 1092182.

[3]. Guyer EC, Ellis EE 3rd, McNamara JA Jr, Behrents RG. Components of class III malocclusion in juveniles and adolescents. Angle Orthod. 1986 Jan;56(1):7-30. PubmedPMID: 3485393.

[4]. Laine T. Associations between articulatory disorders in speech and occlusal anomalies. Eur J Orthod. 1987 May;9(2):144-50. PubmedPMID: 3472893.

[5]. Laine T. Malocclusion traits and articulatory components of speech. Eur J Orthod. 1992 Aug;14(4):302-9. PubmedPMID: 1516663.

[6]. Van Riper C.Speech correction: Principles and methods. New Jersey, Prentice Hall.1978.

[7]. Papageorgiou SN, Gölz L, Jäger A, Eliades T, Bourauel C. Lingual vs. labial fixed orthodontic appliances: systematic review and meta-analysis of treatment effects. European Journal of Oral Sciences. 2016 Apr;124(2):105-18.

[8]. Long H, Zhou Y, Pyakurel U, Liao L, Jian F, Xue J, et al. Comparison of adverse effects between lingual and labial orthodontic treatment. Angle Orthod. 2013 Nov;83(6):1066-73. PubmedPMID: 23581503.

[9]. Rai AK, Rozario JE, Ganeshkar SV. Comparison of speech performance in labial and lingual orthodontic patients: A prospective study. Dent Res J (Isfahan). 2014 Nov;11(6):663-75. PubmedPMID: 25540661.

[10]. Khattab TZ, Farah H, Al-Sabbagh R, Hajeer MY, Haj-Hamed Y. Speech performance and oral impairments with lingual and labial orthodon- tic appliances in the first stage of fixed treatment. Angle Orthod. 2013 May;83(3):519-26. PubmedPMID: 23075062.

[11]. Rai AK, Ganeshkar SV, Rozario JE. Parametric and nonparametric assessment of speech changes in labial and lingual orthodontics: A prospective study. APOS Trends Orthod. 2013 Jul 1;3(4):99-109.

[12]. Atik E, EsenAydinli F, Kulak Kayikçi ME, Ciger S. Comparing the effects of Essix and Hawley retainers on the acoustics of speech. Eur J Orthod. 2017 Aug 1;39(4):440-445. PubmedPMID: 27507127.

[13]. Wan J, Wang T, Pei X, Wan Q, Feng W, Chen J. Speech effects of Hawley and vacuum-formed retainers by acoustic analysis: A single-center randomized controlled trial. Angle Orthod. 2017 Mar;87(2):286-292. PubmedPMID: 27557042.

[14]. Paley JS, Cisneros GJ, Nicolay OF, LeBlanc EM. Effects of fixed labial orthodontic appliances on speech sound production. Angle Orthod. 2016 May;86(3):462-7. PubmedPMID: 26367313.

[15]. Millard T, Richman LC. Different cleft conditions, facial appearance, and speech: relationship to psychological variables. Cleft Palate Craniofac J. 2001 Jan;38(1):68-75. PubmedPMID: 11204685.

[16]. Khan KA, Ibrahim SN, Hamid BA. A Prospective Evaluation of Speech Distortion among Patients Wearing Hawley Retainer Using Acoustics Approach. J Dent Health Oral DisordTher. 2017;6(2):00194.

[17]. Kent RD, ReadC.The Acoustic Analysis of Speech. California, Singular Publishing Group, Inc. 1992.

[18]. Shaw WC. Factors influencing the desire for orthodontic treatment. Eur J Orthod. 1981;3(3):151-62. PubmedPMID: 6943029.

[19]. Guay AH, Maxwell DL, Beecher R. A radiographic study of tongue posture at rest and during the phonation of $/ \mathrm{s} /$ in class III malocclusion. Angle Orthod. 1978 Jan;48(1):10-22. PubmedPMID: 272127.

[20]. O'Gara M, Wilson K. The effects of maxillofacial surgery on speech and velopharyngeal function. ClinPlast Surg. 2007 Jul;34(3):395-402. PubmedPMID: 17692699.

[21]. Scott P, DiBiase AT, Sherriff M, Cobourne MT. Alignment efficiency of Damon3 self-ligating and conventional orthodontic bracket systems: a randomized clinical trial. Am J OrthodDentofacialOrthop. 2008 Oct;134(4):470. e1-8. PubmedPMID: 18929262.

[22]. Rathbone JS, Snidecor JC. Appraisal of speech defects in dental anomalies with reference to speech improvement. The Angle Orthodontist. 1959 Jan;29(1):54-9.

[23]. Kirveskari P, Jaroma M, Laine T. Occlusal adjustment in the treatment of functional voice disorders. Cranio. 1988 Oct;6(4):327-9. PubmedPMID: 3255517. 